\title{
A Facile Fabrication of PA12/CNTs Nanocomposite with Enhanced Three-Dimensional Segregated Conductive Network and Electromagnetic Interference Shielding Property through Selective Laser Sintering
}

\author{
Yu Xiong, Haoran Pei, Qinniu Lv, Yinghong Chen* \\ State Key Laboratory of Polymer Materials Engineering, Polymer Research Institute of \\ Sichuan University, Chengdu 610065, China
}

FTIR measurement was used to investigate the possible molecular interactions in PA12/CNTs system and the results are shown in Figure S1. It can be seen that there are the physical interactions (mostly possible hydrogen bonding) existing between CNTs and PA12 particles. The characteristic absorptions of some functional groups of PA12 can be identified, e.g., $-\mathrm{C}=\mathrm{O}$ group: $1635 \mathrm{~cm}^{-1}$; $-\mathrm{C}-\mathrm{N}$ group: $1537 \mathrm{~cm}^{-1}$; $-\mathrm{N}-\mathrm{H}$ group: $3305 \mathrm{~cm}^{-1}$; $-\mathrm{CH}_{2}$ group: $2925 \mathrm{~cm}^{-1}, 2867 \mathrm{~cm}^{-1}, 3070 \mathrm{~cm}^{-1}$. Comparatively, the profiles of the characteristic absorption bands of PA12, bPA12-CNTs and uPA12-CNTs are similar. However, there are still some differences in the absorption peaks of different samples. After being coated with $\mathrm{CNTs}$, the absorption peaks of $-\mathrm{C}=\mathrm{O}$ group and $-\mathrm{N}-\mathrm{H}$ group of PA12 particles shift from $1635 \mathrm{~cm}^{-1}$ and $3305 \mathrm{~cm}^{-1}$ to $1639 \mathrm{~cm}^{-1}$ and $3307 \mathrm{~cm}^{-1}$, respectively. A bule shift is indicated for $-\mathrm{C}=\mathrm{O}$ group and $-\mathrm{N}-\mathrm{H}$ group absorption, showing that after being coated with CNTs, there is possibly the new hydrogen bonds formed between CNTs and PA12 particles (the strength of the new hydrogen bonds is lower than that of the old PA12 hydrogen bonds)

\footnotetext{
*Corresponding author, E-mail: johnchen@scu.edu.cn 


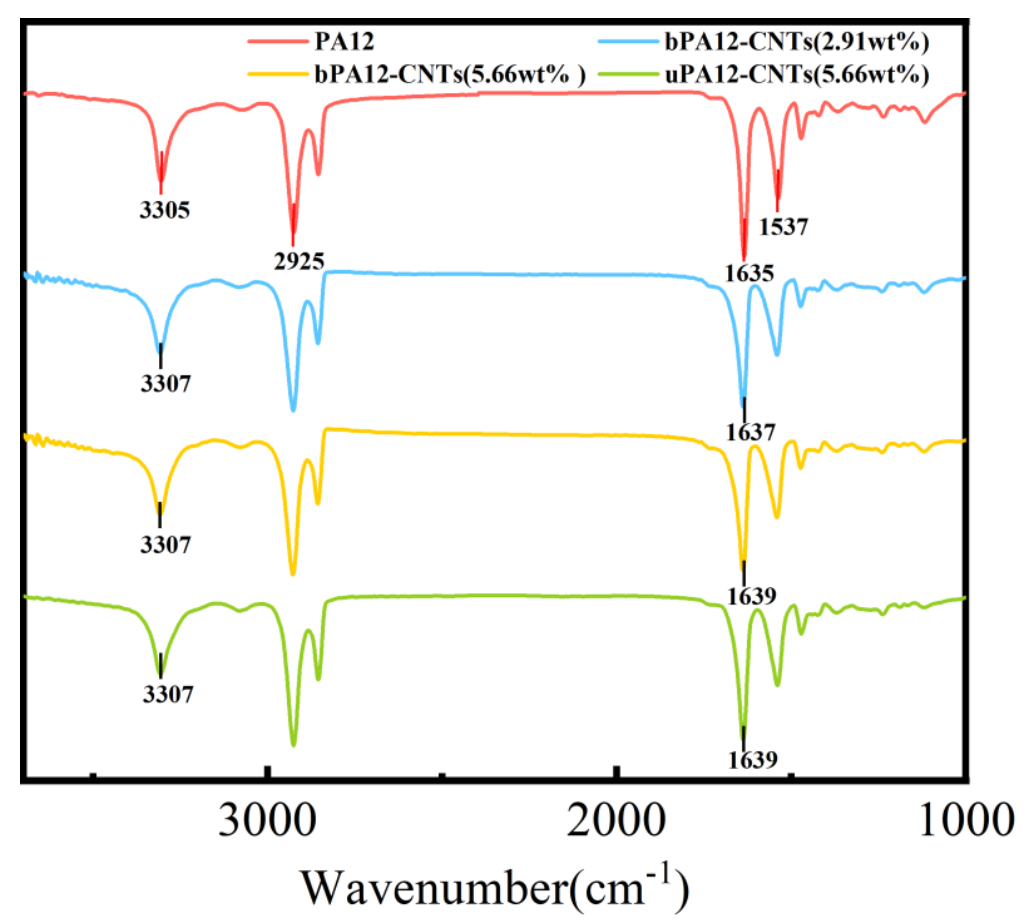

Figure S1 FTIR spectra of different powders: pristine PA12, bPA12-CNTs (2.91 wt $\%$ ) co-powders, bPA12-CNTs (5.66 wt \%) co-powders, uPA12-CNTs (5.66 wt\%) co-powders and mPA12-CNTs $(5.66 \mathrm{wt} \%)$ co-powders.

The particle size of the powder was found to affect the surface quality/accuracy of the SLS-printed part and the density of the final part. Currently, there is no unified conclusion about the optimal particle size distribution for powders used in SLS 3D printing processing. Chung et al ${ }^{1}$. reported that the optimum particle size distribution in terms of processing capability is generally approximately between 10 and $150 \mu \mathrm{m}$. The typical layer thickness in SLS is 0.1-0.2 mm and hence a powder with particle size less than $10 \mu \mathrm{m}$ could make spreading of the powder difficult. Most commercial powders currently used for SLS fall in this range ${ }^{2,3}$. The size and size distribution of composite powders used in this study are listed in Table S1. The span, calculated by the following equation, provides a measurement for the width of particle distribution:

$$
\text { Span }=\frac{D_{90}-D_{10}}{D_{50}}
$$

where $\mathrm{D}_{90}$ is a particle size, below which there are $90 \%$ particles accumulated; $\mathrm{D}_{50}$ is a particle size, below which there are $50 \%$ particles accumulated, i.e., the median particle size; $\mathrm{D}_{10}$ is a particle size, below which there are $10 \%$ particles 
accumulated. As expected, the particle size distributions for all powders are relatively narrow and basically fall in the optimum ranges, which are also mentioned in the above references. But the Span value of mPA12-CNTs is the highest, indicating its particle size distribution is also the widest.

Table S1 The particle size distribution of different composite powders

\begin{tabular}{ccccc}
\hline Sample & $\mathrm{D}_{10}(\mu \mathrm{m})$ & $\mathrm{D}_{50}(\mu \mathrm{m})$ & $\mathrm{D}_{90}(\mu \mathrm{m})$ & Span \\
\hline Pristine PA12 & 42.86 & 58.82 & 76.42 & 0.57 \\
bPA12-CNTs (2.91wt\%) & 41.8 & 57.59 & 84.15 & 0.73 \\
bPA12-CNTs (5.66wt\%) & 37.03 & 56.92 & 86.56 & 0.87 \\
uPA12-CNTs (5.66wt\%) & 37.23 & 58.63 & 99.44 & 1.06 \\
mPA12-CNTs (5.66wt\%) & 25.49 & 86.00 & 155.40 & 1.51 \\
\hline
\end{tabular}

Table S2 The DSC results of pristine PA12 and composite powders

\begin{tabular}{ccccc}
\hline Sample & $\mathrm{T}_{\mathrm{m}}\left({ }^{\circ} \mathrm{C}\right)$ & $\mathrm{T}_{\mathrm{c}}\left({ }^{\circ} \mathrm{C}\right)$ & $\Delta \mathrm{H}_{\mathrm{m}}(\mathrm{J} / \mathrm{g})$ & $\mathrm{X}_{\mathrm{c}}(\%)$ \\
\hline Pristine PA12 & 185.5 & 136.8 & 106.6 & 51.0 \\
bPA12-CNTs (2.91wt $\%)$ & 185.4 & $169.0 / 156.8$ & 97.34 & 46.6 \\
bPA12-CNTs (5.66wt \%) & 184.9 & $169.2 / 158.5$ & 97.28 & 46.5 \\
uPA12-CNTs (5.66wt \%) & 184.5 & $169.4 / 158.6$ & 96.86 & 46.3 \\
mPA12-CNTs (5.66wt\%) & 183.9 & 169.4 & 61.15 & 29.3 \\
\hline
\end{tabular}

The XRD patterns and the crystallinity of bPA12-CNTs $(2.91 \mathrm{wt} \%) 3 \mathrm{D}$ printed part with different energy density and different thickness are shown in Figure S2 and Table S3. As can be seen, for all the samples, there are the similar diffraction curves with two diffraction peaks located at $21^{\circ}$ and $24^{\circ}$, which are corresponding to $\alpha$ crystal and $\gamma$ crystal, respectively. Generally, the $\alpha$ form would be transformed to $\gamma$ form when the sample is heated to the melting temperature ${ }^{4,5}$. As can be seen, the reduction in the peak intensity of $\alpha$ crystal at higher energy density reveals the better melting of PA12 particles, increasing the molten-recrystallization fraction of PA12 being present in $\gamma$ form. In addition, the crystallinity also increases with increasing the laser energy 
density, which contributes to the improvement of mechanical properties. It is known that the XRD peak intensity is sensitive to the thickness of sample. Therefore, the peak intensity also increases while the thickness of sample increases, but the crystal structure and crystallinity are kept unchanged.
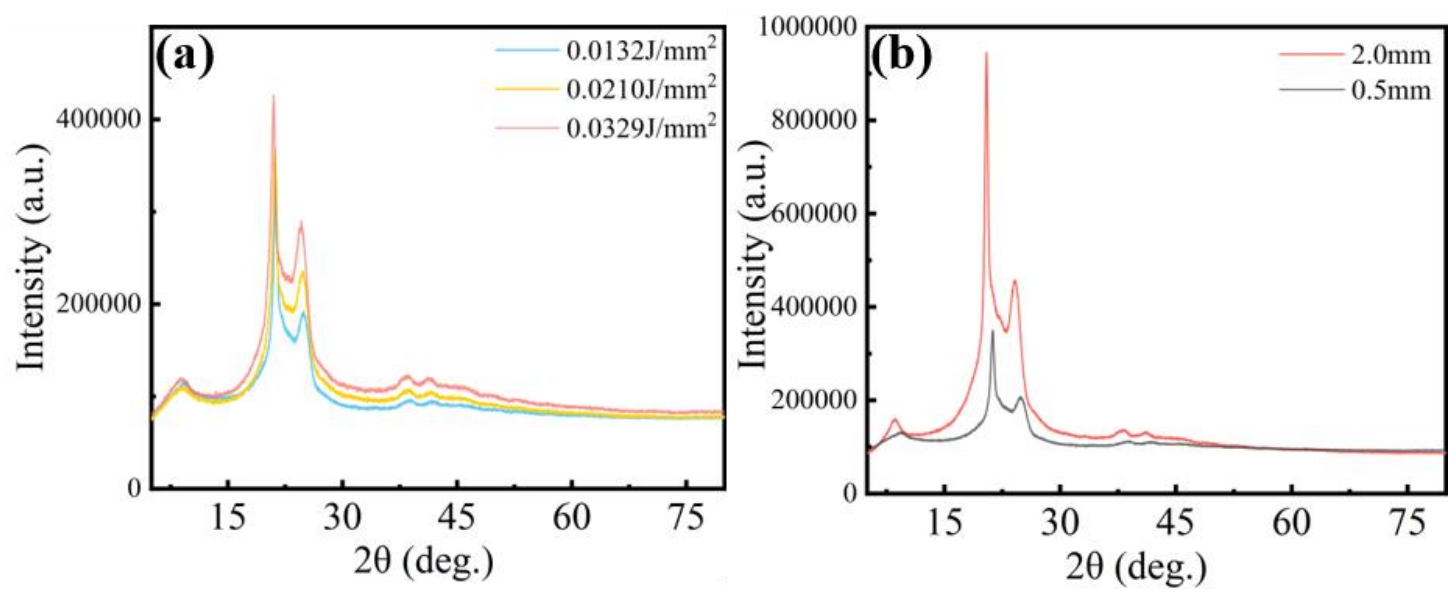

Figure S2 The XRD curves of bPA12-CNTs (2.91 wt $\%$ ) parts at different laser energy density (a) and different thickness (b)

Table S3 The crystallinity of bPA12-CNTs (2.91 wt $\%)$ parts at different parameters and thickness

\begin{tabular}{cc}
\hline Parameter & Crystallinity (\%) \\
\hline $0.0132 \mathrm{~J} / \mathrm{mm}^{2}$ & 29.3 \\
$0.0210 \mathrm{~J} / \mathrm{mm}^{2}$ & 40.6 \\
$0.0329 \mathrm{~J} / \mathrm{mm}^{2}$ & 55.5 \\
$0.5 \mathrm{~mm}$ & 27.5 \\
$2.0 \mathrm{~mm}$ & 29.3 \\
\hline
\end{tabular}




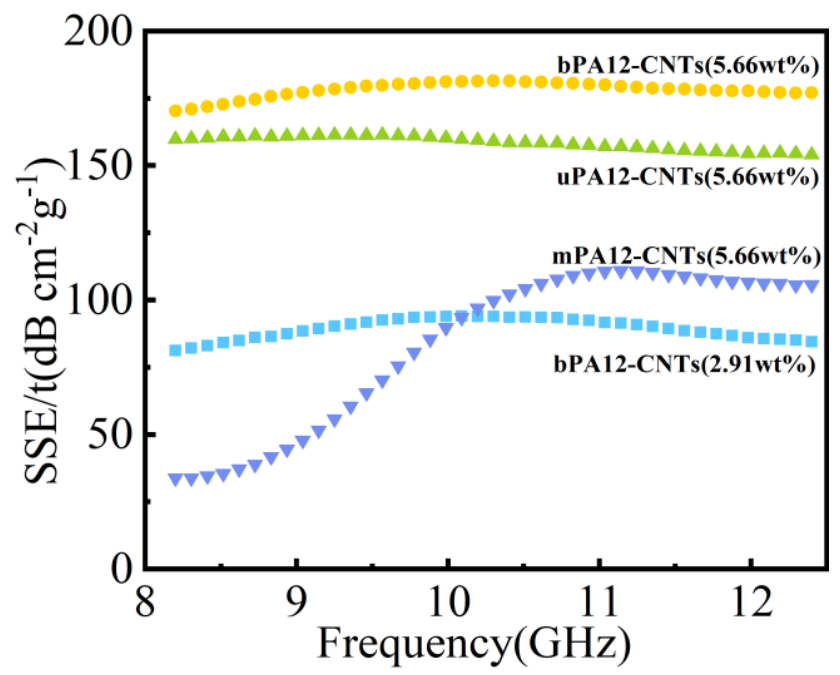

Figure S3 the SSE/t of the PA12/CNTs parts
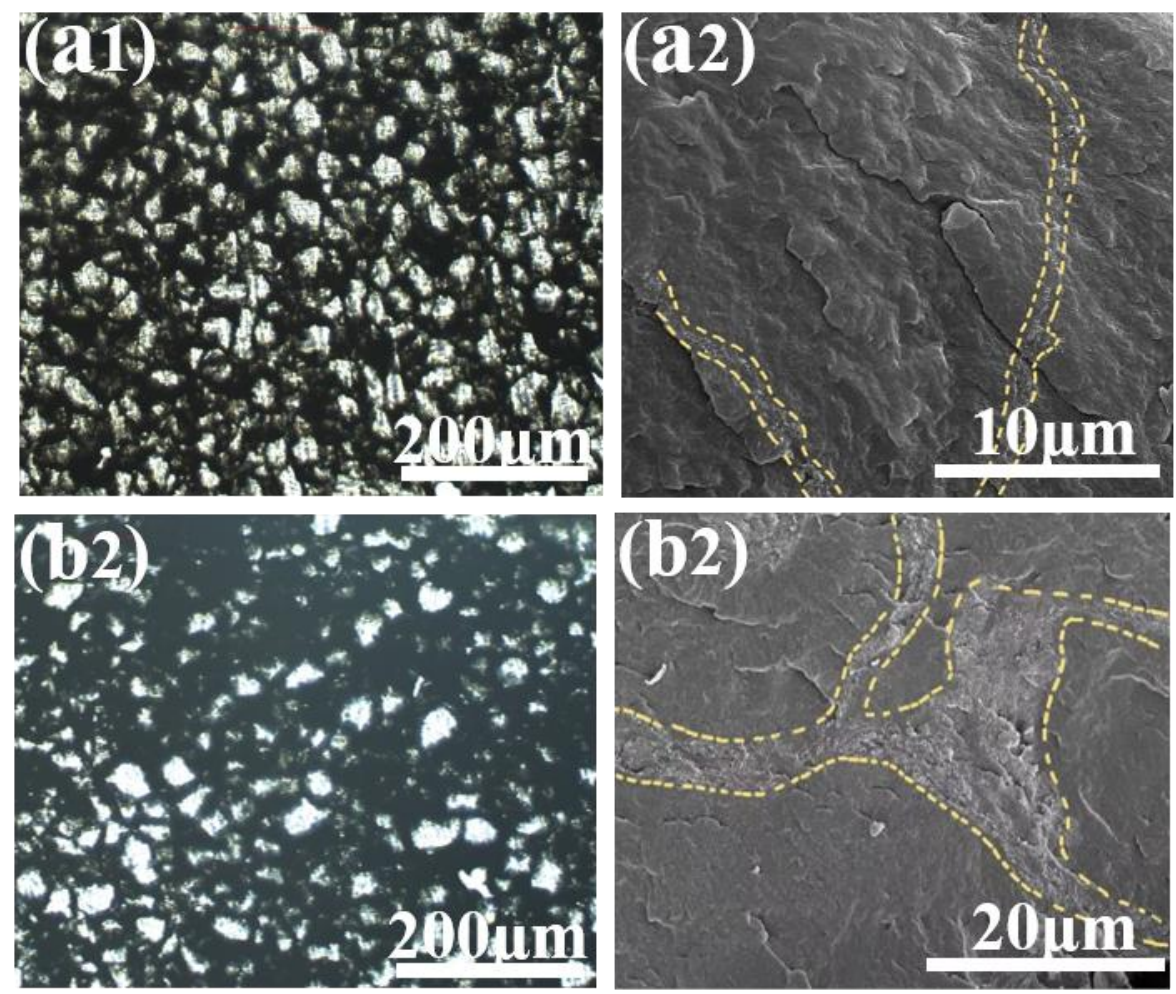

Figure S4 The optical microscopy and SEM photos of the fractured surface of different SLS 3D printed parts: bPA12-CNTs (2.91 wt\%) (a) and uPA12-CNTs (5.66 wt\%) (b)

Figure S6 shows the EMI SE values of PA12/CNTs parts fabricated by different technology. It can be seen that the EMI SE of S-bPA12-CNTs part is much higher than that of M-bPA12-CNTs at the same CNTs loading. For SLS 3D printing method (S-bPA12-CNTs system), the perfect conductive networks (Figure S5a) could be 
formed in the parts due to the weak pressure and shear flow in SLS printing process, which allow the electrons to find more mean free paths and dissipate more electric energies. However, for the melt compounding-compression molding method (M-bPA12-CNTs), it is difficult to form the long-range conductive network in the part, as evidenced by the SEM images (Figure S5b), because the high shear stress occurring in the melt-compounding process could possibly damage the network structures of the conductive fillers. Therefore, the conductive pathways of M-bPA12-CNTs are much shorter than those of SLS printed samples, which is detrimental to the electron movement and thus leads to the decrease in EMI shielding performance. In addition, it is also found that the SSE/t (defined as the SE divided by the thickness and density of the shielding material) of S-bPA12-CNTs part is higher than that of M-bPA12-CNTs part due to the porosity of the S-bPA12-CNTs part.
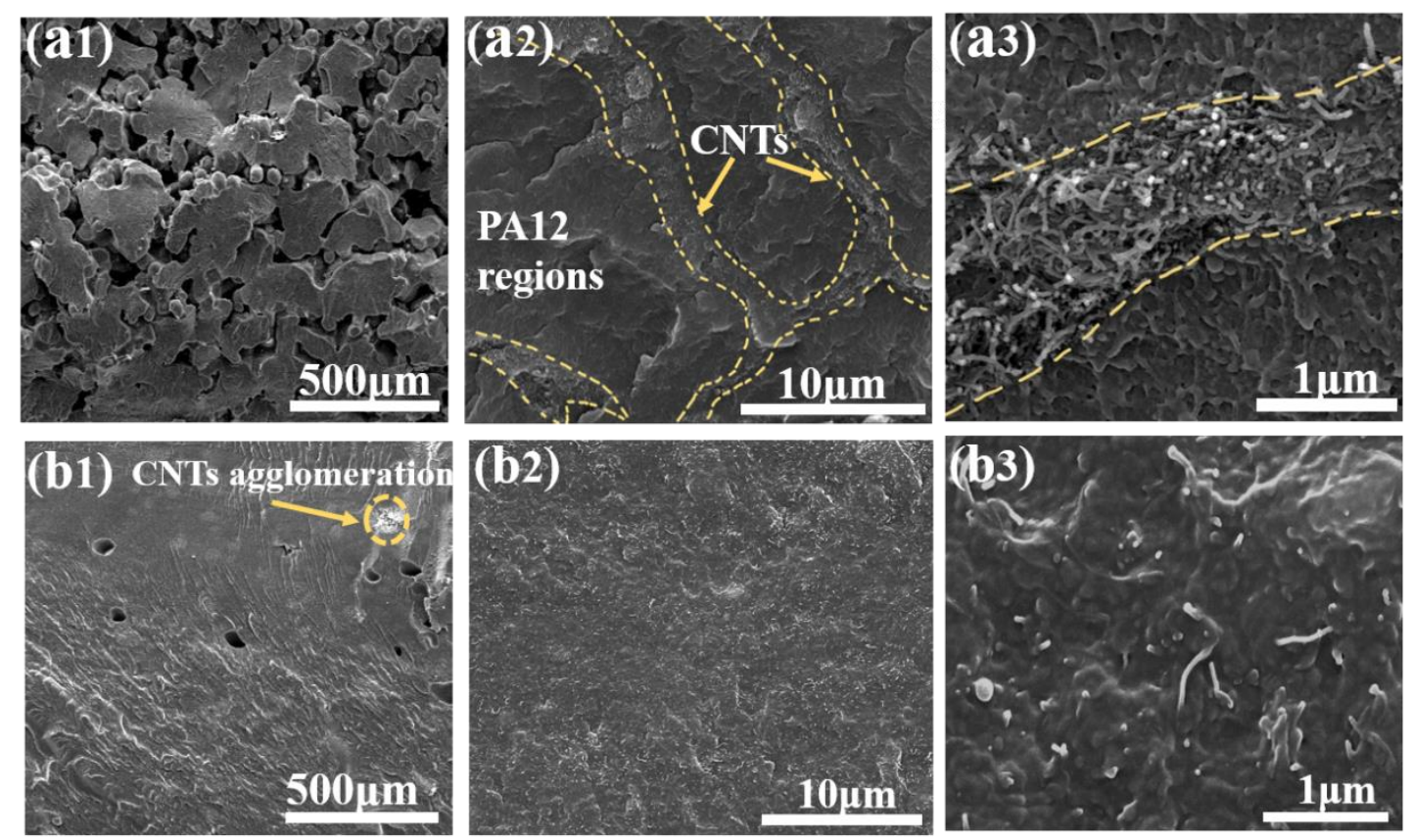

Figure S5 The SEM images of different parts at different magnification: bPA12-CNTs (5.66 wt\%) prepared by SLS (a), bPA12-CNTs (5.66 wt\%) prepared by melt compounding-compression molding (b) 


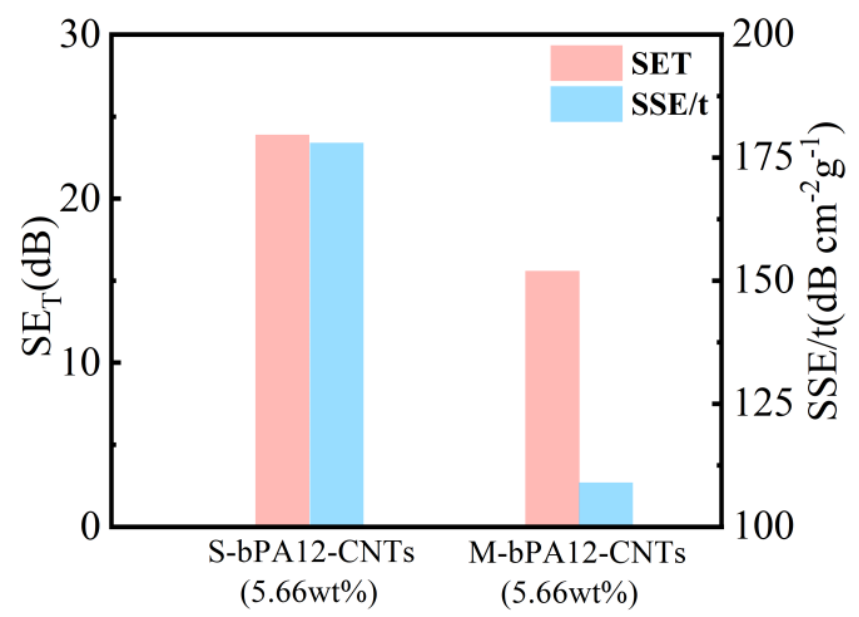

Figure S6 The EMI SET value of different sample: bPA12-CNTs (5.66 wt\%) part prepared via SLS (S-bPA12-CNTs) and bPA12-CNTs (5.66 wt\%) part prepared via melt compounding-compression molding (M-bPA12-CNTs)

Generally, there are two main EMI shielding mechanisms in conductive polymer composites (CPCs), namely reflection and absorption. Reflection occurs due to impedance mismatch between two media which can be expressed by the SER and reflection $(\mathrm{R})$ coefficient. Absorption in CPCs arises from Ohmic loss and polarization loss which can be expressed by the $\mathrm{SE}_{\mathrm{A}}$ and absorption (A) coefficient. In order to investigate the EMI shielding mechanism more deeply and detailedly, the contributions of the reflection (R), absorption (A) and transmission (T) to the total EMI shielding efficiency of different PA12/CNTs nanocomposites were compared (Figure S7).

(a)

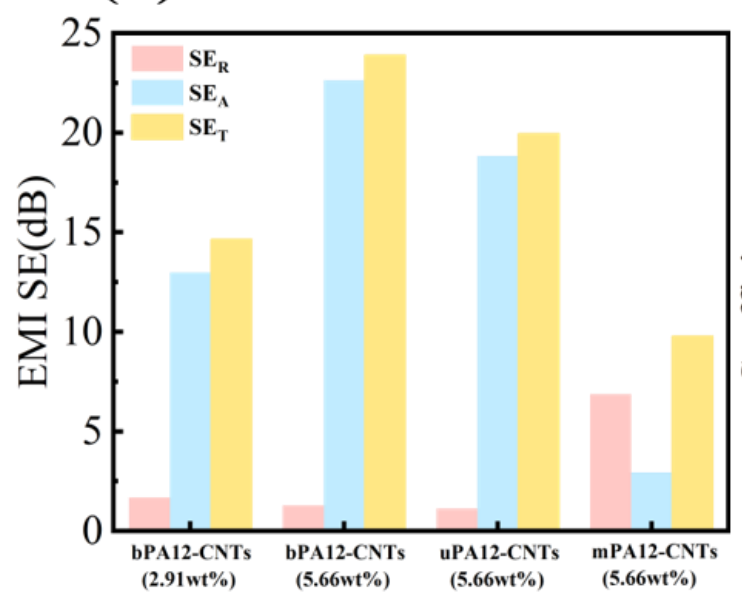

(b)

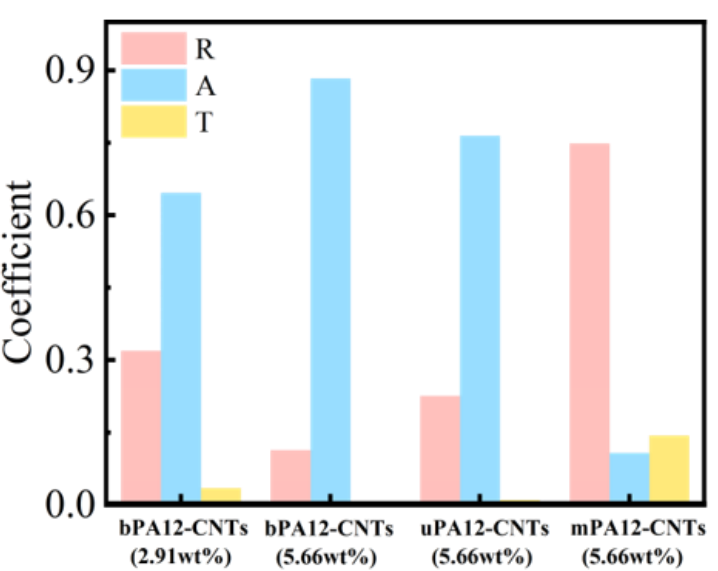

Figure $\mathrm{S} 7 \mathrm{The} \mathrm{SE}_{\mathrm{R}}, \mathrm{SE}_{\mathrm{A}}$ and $\mathrm{SE} \mathrm{E}_{\mathrm{T}}$ values of different 3D printed parts (a); the 
coefficients of the reflection (R), absorption (A) and transmission (T) of different 3D printed parts (b)

The reason why the incorporation of CNTs greatly decreases the MFI value of PA12/CNTs nanocomposite is explained as follows: the incorporated CNTs would restrict the movement of PA12 macromolecular chains though formation of network structures and their strong hydrogen-bonding interactions with PA12 macromolecules and hence the flow of polymer melt. As a result, the incorporation of CNTs would significantly increase the system viscosity. For bPA12-CNTs and uPA12-CNTs co-powders, the CNTs are mostly located on the surface of PA12 particles. The movement of only the local PA12 macromolecular chains on the surface of particles could be hindered. However, for mPA12-CNTs co-powders, the incorporated CNTs are dispersed well in PA12 matrix. Obviously, there are more and more restrictions for the movement of the PA12 macromolecular chains due to the increasing amount of CNTs interacted. This is also the reason why the mPA12-CNTs co-powders have the lower MFI value than the bPA12-CNTs and uPA12-CNTs co-powders at the same CNTs loading. As a result, the content and distribution of CNTs have a significant influence on the PA12/CNTs composite powders.

Figure S8 shows the mechanical performance of different PA12/CNTs nanocomposite parts. It is shown that the M-bPA12-CNTs part exhibits the better mechanical performance than the S-bPA12-CNTs part. One reason for the lower mechanical property of the latter is that in the 3D printed part of S-bPA12-CNTs, there is the interfacial bonding between powder particles which is the common disadvantage of 3D printing technology and would result in reduction in mechanical property. On the other hand, during 3D printing sintering, there are inevitably the pores and defects occurring in the S-bPA12-CNTs parts (Figure S5a), which would become the stress concentration points and thus cause the fracture failure of the material. However, during the compression molding process, there could be almost no pores and defects formed in the parts due to the high pressure. Although the S-bPA12-CNTs part exhibits the lower mechanical property, it could be improved to some extent by optimizing the laser 
energy density and conducting the post-treatment. In the future work, we will be devoted to enhancing the mechanical property of the $3 \mathrm{D}$ printed parts.

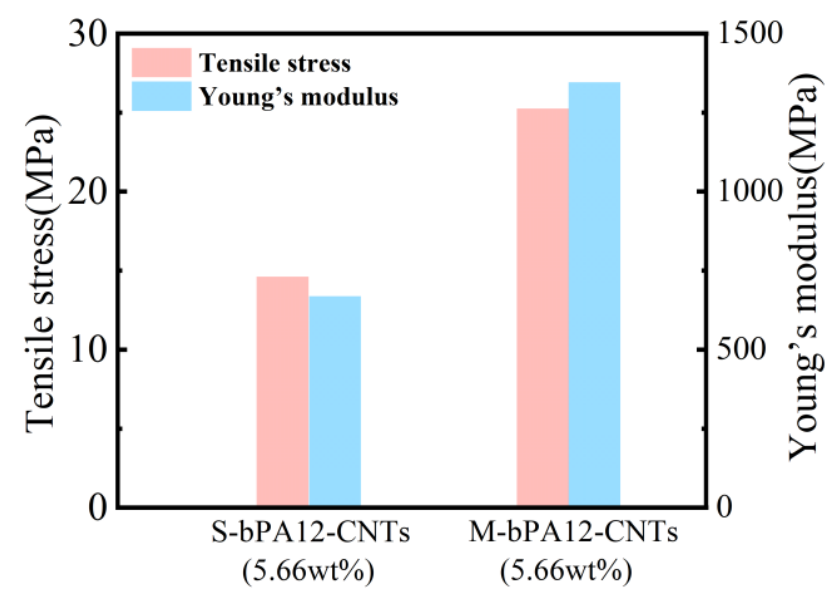

Figure S8 The mechanical performance of different PA12/CNTs nanocomposite part: bPA12-CNTs (5.66 wt\%) part prepared by via SLS (S-bPA12-CNTs) and bPA12-CNTs (5.66 wt\%) part prepared via melt compounding-compression molding (M-bPA12-CNTs)

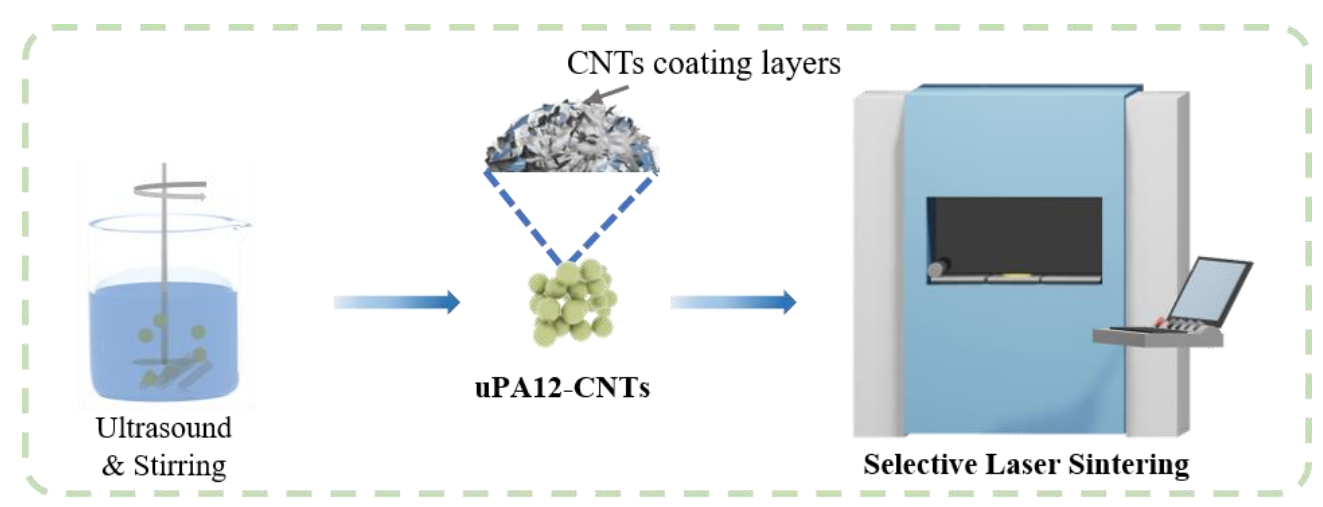

Figure S9 Schematic diagram for preparing ultrasonically treated uPA12-CNTs composite powder

Selective laser sintering is one of the $3 \mathrm{D}$ printing technologies based on the powder system. It relies on the $\mathrm{CO}_{2}$ laser to provide the energy which makes the powder melt and bond to form the final parts. SLS technology possesses the advantages of abundant material selection, non-toxicity, and easy storage. On one hand, the process is simple and does not require additional support structures, which is especially suitable for small batches of personalized customization parts with complex shapes and structures ${ }^{6-8}$. On the other hand, the surface of the SLS 3D printed parts is rough and 
there may be some pores in the parts (Figure 8 in the main text). The picture for the parts of pure PA12 and PA12/CNTs nanocomposite printed by SLS strategy is shown in Figure S10.

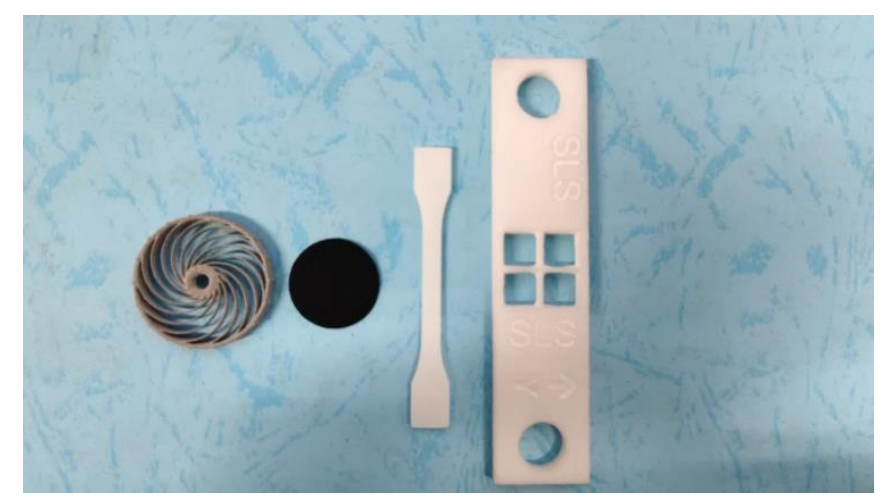

Figure S10 The digital picture for the parts of pure PA12 and PA12/CNTs

nanocomposite printed by SLS strategy

Table S4 The basic parameters for SLS 3D printing

\begin{tabular}{cc}
\hline Parameters & Value \\
\hline Part bed temperature & $177-182^{\circ} \mathrm{C}$ \\
Feed bed temperature & $150^{\circ} \mathrm{C}$ \\
Layer thickness & $0.1 \mathrm{~mm}$ \\
Smart feed gain & 1.8 \\
\hline
\end{tabular}

In order to determine the actual CNTs loading, the TG measurement was carried out. It can be seen that, the amount of the residual CNTs in three kinds of composite powders prepared by different ways is almost the same. The char yield of pure PA12 is near zero. As a result, it is believed that the obtained CNTs loading in different type of PA12/CNTs composite powders is close to the amount of CNTs added. In addition, the initial decomposition temperature increases from 366 to $396{ }^{\circ} \mathrm{C}$ with incorporation of CNTs, indicating that the addition of CNTs could improve the thermal stability of the composite powder. 


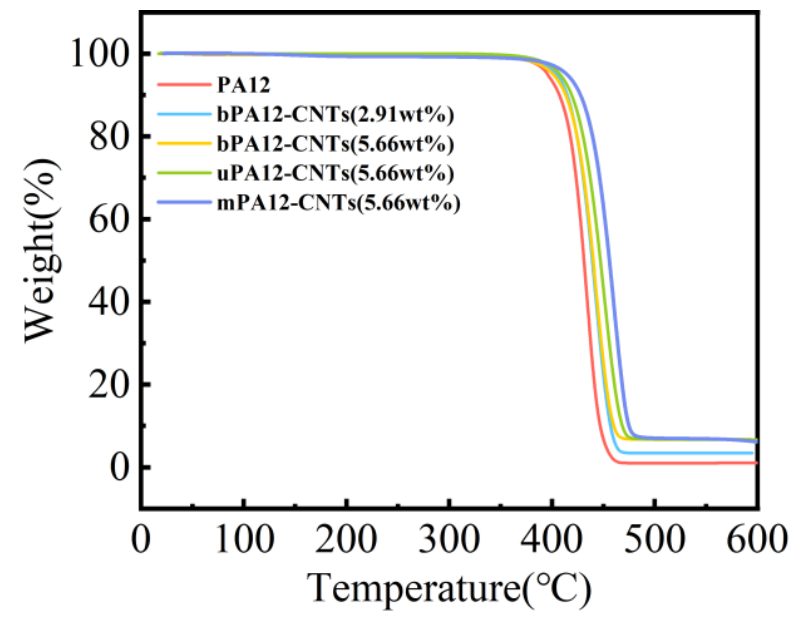

Figure S11 The TGA curve of different composite powders: pristine PA12,

bPA12-CNTs (2.91 wt\%) powders, bPA12-CNTs (5.66 wt\%) powders, uPA12-CNTs

$(5.66 \mathrm{wt} \%)$ powders and mPA12-CNTs $(5.66 \mathrm{wt} \%)$ powders

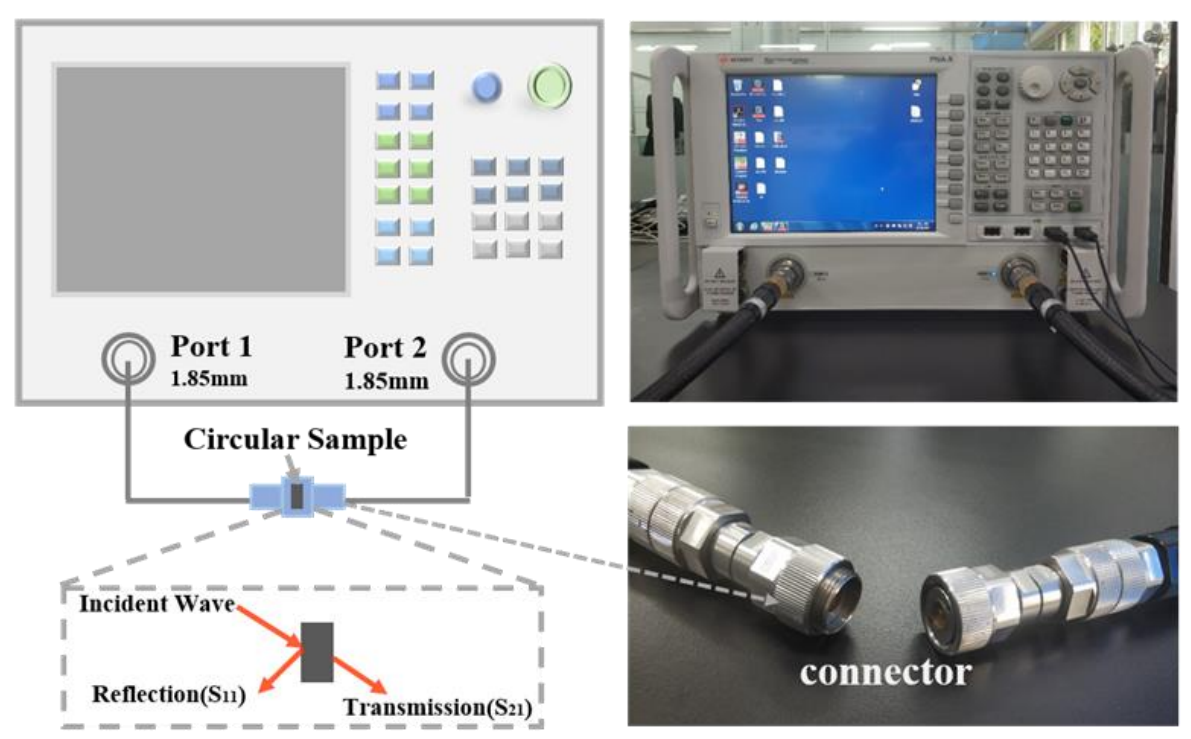

Figure S12 Schematic diagram of EMI shielding measurement setup

\section{REFERENCES}

(1) H. Chung, S. Das, Processing and properties of glass bead particulate-filled functionally graded Nylon-11 composites produced by selective laser sintering. Mater. Sci. Eng. A 2006, 437, 226-234.

(2) L. Verbelen, S. Dadbakhsh, M. Van den Eynde, J.-P. Kruth, B. Goderis, P. Van Puyvelde, Characterization of polyamide powders for determination of laser sintering processability. Eur. Polym. J. 2016, 75, 163-174.

(3) R.D. Goodridge, C.J. Tuck, R.J.M. Hague, Laser sintering of polyamides and other polymers. Prog. Mater. Sci. 2012, 57, 229-267.

(4) Simha Martynkova, G.; Sliva, A.; Kratosova, G.; Cech Barabaszova, K.; Studentova, S.; Klusak, J.; Brozova, S.; Dokoupil, T.; Holesova, S., Polyamide 12 Materials Study of Morpho-Structural Changes during Laser Sintering of 3D Printing. Polymers 2021, 13, 810.

(5) Czelusniak, T.; Amorim, F. L., Influence of energy density on polyamide 12 processed by SLS: 
from physical and mechanical properties to microstructural and crystallization evolution. Rapid Prototyp. J. 2021, 27, 1189-1205.

(6) Yang, L., Tang, Sy., Fan, Zt. et al. Rapid casting technology based on selective laser sintering. China Foundry 2021, 18, 296-306.

(7) Kumar, M. and Sharma, V., "Additive manufacturing techniques for the fabrication of tissue engineering scaffolds: a review", Rapid Prototyp. J. 2021, 27, 1230-1272.

(8) N. Li, D. Qiao, S. Zhao, Q. Lin, B. Zhang, F. Xie, 3D printing to innovate biopolymer materials for demanding applications: A review. Mater. Today Chem. 2021, 20, 100459. 